\title{
Evidence-Based Treatments for Autism Spectrum Disorder
}

Laura C. Politte, M.D. ${ }^{1, *}$

Yamini Howe, M.D. ${ }^{2}$

Lisa Nowinski, Ph.D. ${ }^{2}$

Michelle Palumbo, M.D. ${ }^{2}$

Christopher J. McDougle, M.D. ${ }^{2}$

\author{
Address \\ ${ }^{*}, 1$ Carolina Institute for Developmental Disabilities, University of North Carolina \\ School of Medicine, 101 Renee Lynne Court, Carrboro, NC 27510, USA \\ Email: laura.politte@cidd.unc.edu \\ ${ }^{2}$ Massachusetts General Hospital, Lurie Center for Autism, Harvard Medical School, \\ 1 Maguire Road, Lexington, MA 02421, USA
}

Published online: 30 January 2015

(C) Springer International Publishing AG 2015

This article is part of the Topical Collection on Child and Adolescent Psychiatry

Keywords Autism spectrum disorders - Pervasive developmental disorders - Intellectual disability •

Psychopharmacology · Complementary and alternative medicine $\cdot$ Cognitive behavioral therapy · Dietary

interventions

\section{Opinion statement}

Autism spectrum disorders (ASD) are a heterogeneous group of neurodevelopmental disorders that manifest in early childhood and persist throughout the lifespan; treatment should reflect the unique challenges for that individual at each developmental stage. In early childhood, treatment should focus on the acquisition of language, play skills, joint attention, and effective communication strategies through intensive behavioral and educational interventions, particularly Applied Behavioral Analysis (ABA). Middle childhood and adolescence presents a time for continued skills acquisition, including development of social skills, peer relationships, and maximizing supports for academic weaknesses. In older adolescence and young adulthood, developing vocational and adaptive living skills to maximize opportunities for independence becomes important. ASD are lifelong disorders, and treatment in adulthood includes ensuring opportunities for social, leisure, and vocational activities, maintaining physical health through diet and exercise, and support for transitions in caregiving as parents age. Throughout the lifespan, clinicians should remain mindful of medical complications that can affect behavior and may not be readily apparent in individuals with limited verbal abilities, including gastrointestinal problems such as reflux and constipation, seizures, and allergies. Current pharmacological interventions are primarily aimed at ameliorating the challenging emotional and behavioral symptoms that accompany ASD rather than the core symptoms of ASD themselves. However, substantial evidence-based research into most medications for ASD is 
lacking. Two atypical antipsychotics, risperidone and aripiprazole, have indication for the treatment of severe irritability in youths with ASD, though all other medication use in ASD is considered off-label. Behaviorally based therapies, including ABA and cognitivebehavioral therapy (CBT), may be helpful for symptoms of depression, anxiety, and impaired self-regulation. Clinicians should remain mindful that many families will seek out complementary and alternative medicine (CAM) approaches for their child, and appropriate guidance about the safety and efficacy of these interventions should be offered. Drug therapies that directly target the varied neurobiological underpinnings of ASD are an area of great interest for future research and treatment.

\section{Introduction}

Autism spectrum disorders (ASD) are a group of neurodevelopmental disorders defined by impairments in social communication and patterns of restricted, repetitive behaviors or interests [1]. ASD traits are often apparent in infancy and typically persist across the lifespan, ranging from mild to severe in terms of functional impact. Early deficits in social communication can include diminished eye contact, lack of social smile, decreased responsiveness to name (in the setting of normal hearing), and reduced attempts to establish joint attention (e.g., pointing to objects of interest, referencing a caregiver's response). Children and adults with ASD typically exhibit a reduced range of facial expressions and have difficulty integrating verbal and non-verbal forms of communication. Identifying and interpreting others' emotional states and communicative intent (e.g., joking, sarcasm) can also be challenging. While some individuals with ASD desire to connect with others but lack the social pragmatic skills to do so successfully, others have minimal interest in relationships and prefer solitary activities, making little effort to share their interests. Though no longer considered a core diagnostic feature, many with ASD have language delays, and a minority of individuals never develop functional speech. Characteristic atypical uses of language include echolalia, pronoun reversal, unusual tone of voice, use of scripted phrases, and abnormal prosody. Young children often demonstrate a lack of imaginative play and may prefer repetitive play activities, such as stacking blocks or arranging toys in a particular order. Repetitive behaviors in ASD can include "lower-order" movements, such as stereotypy (e.g., hand flapping, spinning, body rocking) and self-injury, as well as "higher-order" behaviors, such as unusual attachment to objects, insistence on sameness, and restricted interests [2]. Abnormal responses to sensory input, including hypersensitivity and hyposensitivity, are now recognized as characteristic features. "Cognitive rigidity," including resistance to change and difficulty with transitions, is also a hallmark of ASD.

The estimated prevalence of ASD has increased dramatically in the past several years, rising from 1 in 150 children in 2002 to 1 in 68 in $2012[3 \bullet \bullet, 4]$. The precise cause of this sharp rise is unknown, but may include increased awareness and detection of ASD, broader clinical interpretation of the diagnostic criteria, and a true increase due to undetermined environmental factors. Males are 4.5 times more likely to be diagnosed with ASD than females $[3 \bullet \bullet]$. Genetic variations are detected in as many as $30 \%$ of individuals with autism, including 5-7 \% with single-gene disorders (such as tuberous sclerosis and fragile $\mathrm{X}$ syndrome), $5 \%$ with disorders of metabolism, and 7-20\% with copy number variants (CNVs) [5]. Approximately $31 \%$ of individuals with ASD meet criteria for Intellectual Disability (ID; fullscale IQ $\leq 70$ ), and an additional $23 \%$ have cognitive functioning in the borderline range (full-scale IQ 7184) $[3 \bullet \bullet$. Medical co-morbidities in ASD are also common, including seizure disorders in $30 \%$ (with bimodal peak of onset in infancy and adolescence), gastrointestinal problems, and sleep disturbance [6-9].

Treatment across the lifespan requires a multidisciplinary approach that may integrate Early Intensive Behavioral Intervention (EIBI), special education, psychopharmacology, medical interventions, physical therapy, occupational therapy, speech therapy, vocational therapy, social skills training, and instruction in adaptive living skills. Development of language before school age and early joint attention skills are predictive of positive outcomes, and thus a great deal of attention has been focused on early intervention [10-14]. However, the capacity for growth does not end with childhood, and integrated treatment approaches should continue into adulthood. The specific goals of treatment will change depending on the cognitive level and functional abilities 
of the individual with ASD, though interventions should always aim to enhance quality of life, relationships, and degree of independence. In the sections below, we present an overview of current treatment options for the management of both core ASD symptoms and associated clinical features.

\section{Treatment}

\section{Diet and lifestyle}

- Goals for care for individuals with ASD should include consideration of daily life (adaptive) functioning and development of meaningful and functional skills across school, work, home, and community settings. Clinicians should coordinate multidisciplinary evaluations for individuals with ASD, including consultation with developmental and educational specialists, speech/language pathologists, occupational therapists, and physical therapists, in addition to psychiatrists, psy-

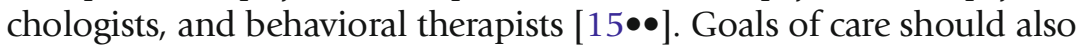
be tailored to consider key times of transition between service models (i.e., Early Intervention, local school department, or state Department of Developmental Services), as well as transition from adolescent to adult services. Families should be counseled regarding disability laws and rights as well as plans for long-term care and guardianship.

- Gastrointestinal (GI) symptoms are common among individuals with ASD, but with a wide range of reported prevalence in studies ranging from 9 to $91 \%$ [16•]. Gluten-free and casein-free (GC/CF) diets are commonly sought by patients based on anecdotal evidence. However, there is an insufficient evidence basis in the medical literature to support the use of a GF/CF diet [17]. GI symptoms should be evaluated by the individual's primary care physician with consideration of referral to a gastroenterologist for further evaluation [7].

- Patients with ASD are often restrictive in the range of foods that they will eat and are therefore at risk for nutritional deficiencies or excess. Consultation with primary care physicians, a nutritionist, or feeding therapists may be needed to expand the range of foods a patient will accept. Calcium intake is of particular concern, but there is limited evidence to support daily vitamin supplementation [18].

- Obesity is common among individuals with ASD, particularly given common use of atypical antipsychotics, and can affect health and quality of life [19].

\section{Pharmacologic treatment}

To date, no medications are approved for the treatment of core symptoms of ASD, including social communication deficits and restricted, repetitive behaviors and interests. Pharmacologic interventions in ASD are primarily aimed at reducing commonly associated symptoms, including inattention, impulsivity, hyperactivity, compulsions, anxiety, sleep disturbance, and irritability-namely severe tantrums, self-injury, and aggression. 


\section{Antidepressant and anxiolytic medications [tricyclic antidepressants (TCAs), selective serotonin reuptake inhibitors (SSRIs), serotonin and norepinephrine reuptake inhibitors (SNRIs), and buspirone]}

Antidepressants have been studied for the treatment of irritability, repetitive behaviors, and compulsions in ASD with mixed results. While some small, open-label trials and randomized trials have suggested a benefit for the agents fluoxetine [20, 21] and clomipramine [22-25] in reducing repetitive behaviors, a relatively large randomized controlled trial (RCT) of citalopram in children failed to show any benefit [26]. A meta-analysis of RCTs of SSRIs for repetitive behaviors in ASD, including unpublished data, suggested a publication bias and no cumulative benefit [27•]. While SSRIs and other antidepressants are rarely helpful in alleviating repetitive behaviors, they may be helpful for treatment of depression and anxiety, which are particularly common in higherfunctioning individuals with ASD. Evidence regarding the treatment of depression and anxiety in ASD is lacking.

If SSRIs and SNRIs (e.g., duloxetine, venlafaxine) are used in children with ASD, start with one fourth to one half of the lowest available dose and titrate slowly (e.g., increase by one fourth to one half tablet every 12 weeks), monitoring for tolerability.

Contraindications

Avoid concurrent use with agents that increase risk for serotonin syndrome.

Avoid concurrent use with monoamine oxidase inhibitors (MAOIs) and other agents that increase risk for serotonin syndrome (e.g., linezolid).

Behavioral side effects with antidepressant use in ASD are common, particularly in pre-pubertal children, and may include irritability, hyperactivity, insomnia, and agitation-symptoms commonly referred to as "behavioral activation" [26]. Other side effects can include nausea, diarrhea, and somnolence. SSRIs are preferred to TCAs due to their greater safety and tolerability profiles. can be effective for the management of anxiety and irritability in ASD [28], though evidence from well-designed RCTs is lacking. Mirtazapine may also cause less behavioral activation than SSRIs and 
can help to regulate sleep disturbances [29]. Mirtazapine can be helpful in the management of problematic sexual behaviors in ASD [30-33]. Increased appetite and weight gain are notable side effects of mirtazapine.

Cost-effectiveness

Generic formulations exist for fluoxetine, sertraline, citalopram, escitalopram, paroxetine, fluvoxamine, mirtazapine, buspirone, duloxetine, and venlafaxine.

\section{Stimulants}

Standard dosage

Symptoms of Attention Deficit-Hyperactivity Disorder (ADHD), including hyperactivity, impulsivity, and inattention, are present in as many as $50 \%$ of individuals with ASD [34]. Methylphenidate (MPH) and mixed amphetamine salt (MAS) formulations may be used in the treatment of ADHD symptoms, though children with ASD are more prone to side effects and show a less robust response rate compared to children with ADHD (49\% vs. $77 \%$ ) $[35,36]$.

Target MPH dose is $1-2 \mathrm{mg} / \mathrm{kg} /$ day; target MAS dose is $0.5-1 \mathrm{mg} / \mathrm{kg} /$ day. Maximum dose reached may be limited by side effects. An ideal dose is reached when therapeutic effects are maximized with a minimum of side effects.

Contraindications

Structural heart disease, arrhythmia, severe or uncontrolled asthma. For individuals with a family history of hypertrophic obstructive cardiomyopathy (HOCM) or sudden death in young people, obtain an electrocardiogram (ECG) before starting.

Main drug interactions

Concurrent use with MAOIs increases the risk for hypertensive crisis.

Main side effects

Lowered appetite, weight loss, headache, stomachache, insomnia, anxiety, irritability, emergence or exacerbation of tics, elevated heart rate and blood pressure.

Special points

If a partial response is obtained but increasing the stimulant dose leads to side effects, consider using in combination with an alpha-2 agonist. Consider short-acting preparations for younger children, who are more prone to side effects, and extended-release preparations for older children and adolescents, to minimize disruptions to the school day. Many capsules can be opened and sprinkled for non-pill swallowers; a MPH transdermal patch and long-acting liquid $\mathrm{MPH}$ formulation are also available. 
Both MPH and MAS are available in generic short-acting and extendedrelease preparations.

\section{Alpha-2 agonists}

Alpha-2 agonists, including guanfacine and clonidine, are often used in the treatment of ADHD symptoms-particularly hyperactivity and impulsivity-and are often better tolerated than stimulants in ASD $[37,38]$. Clonidine is more sedating than guanfacine and can also be used as a sleep aid, though it has a short duration of action (approximately $6 \mathrm{~h}$ ) and is more effective for sleep initiation than for maintenance of sleep.

For guanfacine, start with $0.25-0.5 \mathrm{mg}$ each morning and increase by $0.5 \mathrm{mg}$ weekly in two divided doses up to $4 \mathrm{mg} /$ day, as tolerated. Maximum dosing is $6 \mathrm{mg} /$ day; use three divided doses (TID) for doses $>4 \mathrm{mg} /$ day. For clonidine, start with $0.025 \mathrm{mg}$ TID (or $0.025 \mathrm{mg}$ at bedtime, if used for sleep onset) and increase by $0.025 \mathrm{mg} /$ dose weekly up to $0.2 \mathrm{mg}$ TID, as tolerated. Max dose is $0.6 \mathrm{mg} /$ day. Extended-release preparations of guanfacine and clonidine are also available, though these must be swallowed whole.

\section{Contraindications}

Hypotension. Use with caution in those with structural heart disease or arrhythmia.

Main drug interactions

Avoid concurrent use of agents that lower blood pressure.

Main side effects

Lowered blood pressure, lowered heart rate, sedation, sleep disturbance, constipation, irritability.

Special points

May be used in combination with either stimulants or atomoxetine for partial responders. Initial drowsiness is common, though typically subsides within days. Alpha-2 agonists must be gradually tapered when discontinuing due to risk of rebound hypertension with abrupt cessation.

Guanfacine and clonidine are available in generic forms, though extendedrelease preparations are currently more expensive. 
Atomoxetine

Atomoxetine is a non-stimulant that may be used for associated symptoms of ADHD in ASD. Improvements in hyperactivity are generally more robust than improvements in inattention, and response rates are similar to those reported in studies of stimulants in ASD, though effect sizes are more modest [39, 40].

Standard dosage

Recommended dosing is approximately $1.2 \mathrm{mg} / \mathrm{kg} /$ day, up to a maximum of $100 \mathrm{mg} /$ day. A cautious titration schedule is recommended for most, starting with $10 \mathrm{mg}$ every morning and increasing by $10 \mathrm{mg}$ weekly to maximum tolerated dose. May be dosed twice daily to minimize side effects or if a "wearing off" effect occurs later in the day with once daily dosing.

Contraindications

Use with caution in individuals with structural heart disease or arrhythmias.

Main drug interactions

Concurrent use with MAOIs increases risk for serotonin syndrome.

Main side effects

Somnolence, nausea/gastrointestinal upset, headache, sleep disturbance, lowered appetite, increased heart rate, increased blood pressure, and irritability.

Special points

As with stimulants, children with ASD appear to have a more modest therapeutic response to atomoxetine than children with ADHD without ASD [40]. Higher-functioning children with ASD show higher response rates compared with their peers with more severe autistic symptoms or ID $[41,42]$.

Cost-effectiveness

Atomoxetine is not yet available in generic form and is more expensive than generic stimulant, guanfacine, and clonidine preparations.

\section{Atypical antipsychotics}

Atypical antipsychotics are primarily used for the treatment of severe irritability in ASD, including mood lability, severe tantrums, aggression, and self-injury. Based on the results of relatively large RCTs, risperidone and aripiprazole are the only two medications specifically approved by the Food and Drug Administration (FDA) for treatment of severe irritability in children with ASD (for ages 5-16 years old for risperidone and 6-17 years old for aripiprazole) [43-46]. The potential benefits of these 
medications must be weighed against their side effect profile, which can include weight gain and metabolic derangement. Atypical antipsychotics are preferred over first-generation antipsychotics for their lower risk of extrapyramidal symptoms (EPS) and tardive dyskinesia (TD).

Varies by agent. For risperidone, start with $0.25-0.5 \mathrm{mg}$ at bedtime and increase by $0.25-0.5 \mathrm{mg}$ daily each week up to a maximum of $4 \mathrm{mg} /$ day; many patients will have a therapeutic response at a lower dose. May be given once daily or in two to three divided doses. For aripiprazole, start with $2.5 \mathrm{mg}$ in the morning or at bedtime and increase to $2.5 \mathrm{mg}$ each week up to a maximum of $20 \mathrm{mg} /$ day; most patients will have a therapeutic response at a lower dose. May be given once daily or in two divided doses.

Contraindications

No absolute contraindications, though should be used with caution in elderly patients due to increased risk of death associated with antipsychotic use in this population.

Main drug interactions

Concurrent use with metoclopramide increases the risk of extrapyramidal symptoms and neuroleptic malignant syndrome (NMS). Avoid concurrent use with medications that also cause prolongation of the QT interval (e.g., pimozide, cisapride, select antibiotics).

Main side effects

Increased appetite, weight gain, somnolence, fatigue, muscle stiffness, EPS, drooling, QT interval prolongation, elevated cholesterol, elevated fasting blood glucose, nausea and vomiting. All atypical antipsychotics, aside from aripiprazole, are associated with elevated prolactin levels, which can lead to menstrual irregularities and galactorrhea in females as well as gynecomastia and galactorrhea in males. Ziprasidone tends to be weight-neutral, though is associated with a greater degree of QT prolongation, and EKGs should be obtained at baseline, with dosage changes, and every 6-12 months during maintenance treatment. For all agents, fasting glucose and lipid panels should be obtained at baseline and then every 6-12 months thereafter.

Before beginning an antipsychotic for irritability, a careful medical evaluation should be done to rule out physical discomfort as a source of agitation. Similarly, environmental factors that may be contributing to or maintaining maladaptive behavior patterns should first be identified and modified through behavioral interventions. Some parents find that when their children gain weight while taking antipsychotics, they become more difficult to manage physically when agitated. 
Cost-effectiveness

Generic preparations are available for risperidone, olanzapine, quetiapine, and ziprasidone.

\section{Complementary and alternative medicine (CAM)}

- Up to $50 \%$ of families with children with ASD use CAM treatments at some point, with higher rates among those with GI symptoms, seizures, and behavioral problems $[47,48]$. Despite their frequent use, clinical trials are lacking to support or refute most CAM interventions, both ingestible and non-ingestible [49•].

- Clinicians should assist families in weighing the safety profile, ease of use, degree of evidence, and expense associated with CAM treatments under consideration.

- Despite initial enthusiasm for secretin based on positive case reports, intravenous secretin therapy for treatment of ASD symptoms has not been found effective in more than 16 RCTs and is not recommended [50].

- Melatonin has been shown to improve sleep onset in numerous controlled trials and is currently recommended as a first-line medication for initial insomnia in ASD [51]. Melatonin is available over-thecounter in a number of different preparations. A typical starting dose is $3 \mathrm{mg}$ given $1 \mathrm{~h}$ before bedtime, increasing by $3 \mathrm{mg}$ every week up to $9 \mathrm{mg} /$ day as needed. Younger children may start with 1-2 $\mathrm{mg}$ with smaller dosing increments.

- Multivitamin supplementation can be considered for individuals with limited or poor diets [49•].

\section{Interventional procedures}

\section{Hyperbaric oxygen therapy}

- Hyperbaric oxygen therapy (HBOT) is proposed to improve oxygen levels in the body, which leads to decreased inflammation and less oxidative stress. There has been some evidence to suggest increased levels of inflammation, oxidative stress, and abnormalities in inflammatory markers, such as cytokines, in individuals with ASD, thus, leading to the hypothesis that HBOT may improve these abnormalities with resultant improvement of ASD symptoms [52].

- The evidence for the efficacy of HBOT is limited to multiple case series, an open-label study, and a few controlled trials, three of which were double-blind and placebo-controlled. Only one of the randomized placebo-controlled trials had a positive significant finding [53]. The findings of the other RCTs did not support the earlier finding [54, 55]. In a different study, changes in cytokine levels were not associated with 
a positive response to HBOT [52].

- In the studies with ASD populations, HBOT was well tolerated; however, known complications from HBOT in other disorders include CNS oxygen toxicity, which can cause seizures, and barotrauma, which can lead to tympanic membrane rupture. At this time, the use of HBOT for the treatment of ASD is not recommended.

\section{Chelation therapy}

- Heavy metal exposure, namely mercury, has been proposed as a cause for the increased prevalence of ASD. Chelation therapy is approved for detoxification of heavy metals from the body in documented cases, such as in lead poisoning.

- Strong evidence of chelation's benefit for the treatment of ASD is lacking. In 2007, the NIMH halted a clinical trial after animal models demonstrated "lasting cognitive impairment" [56]. Furthermore, there have been deaths reported secondary to hypocalcemia from intravenous chelation using the agent EDTA $[57,58]$. As such, chelation therapy is not recommended.

\section{Electroconvulsive therapy for catatonia}

- ECT is a highly effective treatment in disorders such as major depression and catatonia in typical developing individuals. The majority of the evidence for ECT in catatonia in ASD is limited to case reports and small case series. Published results so far have been positive. Larger controlled trials are warranted [59].

- Individuals with ASD often have language impairments and may not develop the ability to communicate using speech. Frustration with communication can cause or exacerbate behavioral problems, such as aggression, self-injurious behaviors, or chronic irritability. Alternative and Augmentative Communication (AAC) systems and protocols have been developed to aid in language acquisition and can serve as a means of communication in individuals with limited or no verbal language abilities. Careful evaluation by a speech-language pathologist experienced in the use of AAC methods for individuals with ASD is recommended.

- In children, the Picture Exchange Communication System (PECS) is widely used and has been shown to be effective in some controlled trials and observational studies, particularly when used in early childhood [60].

- Technological breakthroughs with touch screen handheld devices have led to rapid development of software and speech-generating devices 
[61]. Evidence for efficacy of AAC devices for minimally verbal children is emerging [62]

\section{Physical, speech, and other therapies}

Although many of the primary interventions for ASD involve behavioral treatment, several adjunctive therapies can be instrumental in treating the related language, motor, and sensory challenges inherent in ASD.

- Speech Therapy: Individuals with ASD present with a variety of language and communication challenges across the lifespan. Speech therapy is often an integral intervention aimed to address the myriad of speech and language concerns experienced by individuals with ASD, including delayed language development, impaired articulation, and limited social pragmatics and social communication skills. Speech therapy has been found effective in increasing the use of spontaneous speech in individuals with ASD [63]. In addition, speech therapy has been found to improve overall conversational competence, parentrated pragmatic functioning and social communication, and teacherrated classroom learning skills for school-age children with ASD [64].

- Occupational Therapy: Many individuals with ASD also experience delays and ongoing deficits in gross motor coordination and fine motor skill $[65,66]$. Occupational therapy can be effective in remediating the gross and fine motor challenges in individuals with ASD and has even been found to result in gains in early cognitive development [67].

- Sensory Integration Therapy: Separate, but related to an individual's need for OT, some individuals with ASD present with sensory processing differences that require specific intervention. In fact, atypical sensory processing is now included as a diagnostic subcriterion in the DSM-5. Although the empirical support for sensory integration therapy is limited [68], sensory integration approaches are often utilized in occupational therapy for individuals with ASD [69].

\section{Other treatments (behavioral, educational, and psychosocial interventions)}

- Applied Behavior Analysis (ABA) and Discrete Trial Training (DTT): There is a substantial body of literature that supports the use of early, intensive behavioral interventions for children with ASD [70-72]. ABA, or DTT, is one of the most common forms of behavior therapy and is based on the behavior analysis. Complex behaviors are broken down into smaller, "discrete" skills and taught in a systematic fashion over time using substantial repetition and reinforcement [73]. 
- Pivotal Response Training (PRT): Building on basic models of ABA, PRT focuses explicitly on "pivotal" areas of learning and development, including an individual's motivation and response to cues, in order to build new skills and behaviors. A focus on natural reinforcers replaces the use of the tangible and edible reinforcers in DTT [74, 75].

- Floortime: Based in the Developmental Individual Difference Relationship-based (DIR) model, Floortime is a framework for early intervention that focuses on a child's broad social-emotional development, rather than isolated or discrete skills. Intervention is primarily play-based and focuses on activities that are appropriate to a child's developmental level and interests [76, 77].

- Early Start Denver Model (ESDM): Combining elements of structured ABA with more informal "relationship-based" approaches to intervention, the ESDM pulls from the therapeutic methods described above and has been found to reduce ASD symptom severity over time [78, $79 \bullet \bullet$ ].

- Special Education Services: Many children with ASD require substantial support in their educational programming. An Individualized Education Program (IEP) is often an essential part of planning and monitoring a student's appropriate academic progress. Students can be placed on an IEP as early as 3 years old and many remain in formal school programming through their 22nd birthday.

- Treatment and Education of Autistic and Related Communication (TEACCH): The TEACCH model is a set of teaching and intervention strategies that is based on the specific learning needs of an individual with ASD, including strength in visual processing, reduced executive functioning skills, and difficulties with social communication. Emerging research suggests some positive effects on perceptual, motor, verbal, and cognitive skill development, as well as improved social skills and behavior [80].

- Parent Management Training: Parent management training has been found effective in reducing parental stress and improving management of problematic behaviors such as aggression, non-compliance, and selfinjury in children with ASD [81-85]. In addition, the combination of parent training and medication has been found to reduce serious maladaptive behaviors more than medication alone, and in some cases even allows for a lower medication dose [86].

- Cognitive Behavioral Therapy (CBT): Research suggests that CBT is effective in reducing concurrent psychiatric symptoms, such as anxiety, in individuals with ASD [87•]. 


\section{Emerging therapies}

- Family Therapy: Parents of children with ASD often experience increased psychological distress, marital problems, and family conflict $[88 \bullet, 89,90]$. Family therapy, including couples counseling, may be helpful in supporting the overall well-being of a family system.

\section{Oxytocin}

- Oxytocin is a peptide hormone that is responsible for labor contractions, lactation, and more recently has been associated with the development of social behaviors. Oxytocin and its effects have been studied in multiple disorders, including depression, anxiety, personality disorders, schizophrenia, and ASD. Low oxytocin levels have been identified in some individuals with ASD, and social impairment in ASD may be due to dysfunction within the oxytocin system in a subset of individuals.

- Multiple studies have demonstrated oxytocin's effects on social motivation, social initiation, social cognition, and repetitive behaviors in ASD [91]. Small clinical trials and other small studies have been completed at this time with overall mixed results; larger clinical studies are currently in progress [91-93].

- The use of oxytocin for the treatment of ASD is not recommended at this time.

- Arbaclofen is a GABA-B agonist that is related to the muscle-relaxing agent, baclofen. In animal models, arbaclofen has been demonstrated to decrease anti-social and repetitive behaviors. Studies in patients with fragile X syndrome have shown similar findings.

- A small, open-label trial in idiopathic ASD demonstrated improvements in irritability and social withdrawal scales, obsessive-compulsive symptoms, and social responsiveness [93]. Side effects of the medication included agitation, irritability, fatigue, increased motor activity, insomnia, and diarrhea.

- Due to lack of substantive evidence from RCTs, the use of arbaclofen for the treatment of ASD is not recommended at this time [94].

- Memantine is an FDA-approved medication for the treatment of Alzheimer's disease. Memantine is an NMDA antagonist that modulates glutamate release. Glutamate toxicity has been cited as a proposed mechanism for ASD [95].

- Multiple case reports, open-label studies, and one randomized controlled trial of memantine suggest a positive effect for social communication deficits. Side effects reported included abdominal pain, appetite changes, dizziness, insomnia, nausea, sedation, worsening of 
behaviors, irritability, and rash [96]. Dosages in the studies ranged from 2.5 to $30 \mathrm{mg}$ per day.

- Further studies are required at this time. Currently, the use of memantine is not recommended.

- N-Acetylcysteine (NAC) has antioxidant properties and modulates glutamate. The results of two small RCTs are promising in terms of reducing autism-related irritability. In both trials, NAC was well tolerated with mild side effects of gastrointestinal symptoms, increased appetite, nervousness, fatigue, and daytime sedation [97, 98].

- The use of NAC for the treatment of ASD is not recommended at this time due to lack of substantive evidence.

\section{Pediatric considerations}

- Early intensive behavioral treatment programs are well established as the treatment of choice for children with ASD and have been shown to improve outcomes significantly [99]. Given that intervention services before diagnosis are limited, early identification and referral to treatment services is key to improving outcomes. The American Academy of Pediatrics (AAP) has recommended routine surveillance for ASD at every well-child visit, standardized developmental screenings at ages 9, 18 , and 30 months, and ASD-specific screenings at ages 18 and 24 months [100].

- Routine health care is important for individuals with ASD, as with all individuals. However, those with ASD may be more likely to have medical conditions relating to etiology, such as fragile X syndrome or tuberous sclerosis, that affect health care needs. Specific repetitive behaviors can affect health, and clinicians should be alert to these symptoms. For example, repeated skin picking or rubbing can lead to skin infection, and pica or mouthing behaviors increase the risk of lead exposure. Furthermore, routine health visits can be difficult due to behavioral, sensory, and communication challenges. Clinicians should be alert to these needs and help individuals access routine dental and medical care. Accommodations, such as slowing the pace of the visit and using visual supports and stories, may be used [101]. Specific behavioral goals for treatment planning might include learning to tolerate blood pressure checks and blood draws, and learning to swallow pills. 


\section{Conclusion}

- Use of psychotropic medications is common among children and adolescents with ASD [102•]. However, given the limited evidence basis, variable efficacy, and high rate of side effects for psychotropic medications, the common adage "start low and go slow" should be followed when prescribing psychotropic medications for children and adolescents with ASD.

ASD are complex neurodevelopmental disorders that require a multidisciplinary treatment approach across the lifespan. The greatest strength of evidence for treatment of core social communication impairments in ASD exists for behavioral interventions, particularly when implemented early in development. Pharmacological interventions may be helpful in alleviating associated emotional and behavioral symptoms, though a careful medical evaluation must first be conducted to rule out physical sources of discomfort or other etiologies for maladaptive behaviors (such as seizures). Novel treatments that target specific neurobiological processes in ASD and are effective for core deficits of the disorder are an important area of focus for future research.

\section{Acknowledgment}

This manuscript was funded, in part, by the Nancy Lurie Marks Family Foundation.

\section{Compliance with Ethics Guidelines}

\section{Conflict of Interest}

Laura C. Politte declares that she has no conflict of interest.

Yamini Howe declares that she has no conflict of interest.

Lisa Nowinski declares that she has no conflict of interest.

Michelle Palumbo declares that she has no conflict of interest.

Christopher McDougle declares that he has no conflict of interest.

\section{Human and Animal Rights and Informed Consent}

This article does not contain any studies with human or animal subjects performed by any of the authors. Among cited articles where one of the authors of the current report were authors, local Institutional Review Board approval was obtained and maintained for studies where human (or animal) subjects research was performed. 


\section{References and Recommended Reading}

Papers of particular interest, published recently, have been

highlighted as:

- Of importance

$\bullet$ Of major importance

1. American Psychiatric Association, editor. Diagnostic and statistical manual of mental disorders: DSM-5. 5th ed. Washington: American Psychiatric Association; 2013.

2. Turner M. Annotation: repetitive behaviour in autism: a review of psychological research. J Child Psychol Psychiatry. 1999;40(6):839-49.

3.• Prevalence of autism spectrum disorder among children aged 8 years-autism and developmental disabilities monitoring network, 11 sites, United States, 2010. MMWR Surveill Summ. 2014;63(2):1-21.

This article summarizes the latest findings of CDC regarding increasing prevalence of ASD.

4. Prevalence of autism spectrum disorders-autism and developmental disabilities monitoring network, 14 sites, United States, 2002. MMWR Surveill Summ. 2007;56(1):12-28.

5. Schaaf CP, Zoghbi HY. Solving the autism puzzle a few pieces at a time. Neuron. 2011;70(5):806-8.

6. Depositario-Cabacar DF, Zelleke TG. Treatment of epilepsy in children with developmental disabilities. Dev Disabil Res Rev. 2010;16(3):239-47.

7. Buie $\mathrm{T}$ et al. Recommendations for evaluation and treatment of common gastrointestinal problems in children with ASDs. Pediatrics. 2010;125 Suppl 1:S1929.

8. Hollway JA, Aman MG. Sleep correlates of pervasive developmental disorders: a review of the literature. Res Dev Disabil. 2011;32(5):1399-421.

9. Kotagal S, Broomall E. Sleep in children with autism spectrum disorder. Pediatr Neurol. 2012;47(4):24251.

10. Anderson DKet al. Patterns of growth in verbal abilities among children with autism spectrum disorder. J Consult Clin Psychol. 2007;75(4):594-604.

11. Howlin P, Mawhood L, Rutter M. Autism and developmental receptive language disorder-a follow-up comparison in early adult life. II: Social, behavioural, and psychiatric outcomes. J Child Psychol Psychiatry. 2000;41(5):561-78.

12. Mawhood L, Howlin P, Rutter M. Autism and developmental receptive language disorder-a comparative follow-up in early adult life. I: Cognitive and language outcomes. J Child Psychol Psychiatry. 2000;41(5):547-59.

13. Wodka EL, Mathy P, Kalb L. Predictors of phrase and fluent speech in children with autism and severe language delay. Pediatrics. 2013;131(4):e1128-34.

14. Weitlauf AS, et al. Therapies for Children With Autism Spectrum Disorder: Behavioral Interventions Update 2014, Rockville MD.
15.• Volkmar F et al. Practice parameter for the assessment and treatment of children and adolescents with autism spectrum disorder. J Am Acad Child Adolesc Psychiatry. 2014;53(2):237-57.

This article presents most current AACAP guidelines regarding diagnosis and management of autism spectrum disorders.

16. Coury DL, et al. Gastrointestinal conditions in children with autism spectrum disorder: developing a research agenda. Pediatrics 2012: United States. p. S160-8.

This article presents review of the literature and current state of knowledge regarding GI symptoms in ASD. This article also identifies areas needing further investigation, presented based on expert panel symposium. This article was published in a supplement to Pediatrics with many other key article summarizing current state of research related to health care for children with ASD.

17. Mari-Bauset S., et al. Evidence of the gluten-free and casein-free diet in autism spectrum disorders: a systematic review. J Child Neurol. 2014.

18. Hyman SL et al. Nutrient intake from food in children with autism. Pediatrics. 2012;130 Suppl 2:S145-53.

19. Curtin C, Jojic M, Bandini LG. Obesity in children with autism spectrum disorder. Harv Rev Psychiatry. 2014;22(2):93-103.

20. Hollander E et al. A placebo controlled crossover trial of liquid fluoxetine on repetitive behaviors in childhood and adolescent autism.

Neuropsychopharmacology. 2005;30(3):582-9.

21. Hollander E et al. A double-blind placebo-controlled trial of fluoxetine for repetitive behaviors and global severity in adult autism spectrum disorders. Am J Psychiatry. 2012;169(3):292-9.

22. Brasic JR et al. Clomipramine ameliorates adventitious movements and compulsions in prepubertal boys with autistic disorder and severe mental retardation. Neurology. 1994;44(7):1309-12.

23. Brodkin ES et al. Clomipramine in adults with pervasive developmental disorders: a prospective open-label investigation. J Child Adolesc Psychopharmacol. 1997;7(2):109-21.

24. Gordon CT et al. A double-blind comparison of clomipramine, desipramine, and placebo in the treatment of autistic disorder. Arch Gen Psychiatry. 1993;50(6):441-7.

25. McDougle CJ et al. Clomipramine in autism: preliminary evidence of efficacy. J Am Acad Child Adolesc Psychiatry. 1992;31(4):746-50.

26. King $\mathrm{BH}$ et al. Lack of efficacy of citalopram in children with autism spectrum disorders and high levels of repetitive behavior: citalopram ineffective in children with autism. Arch Gen Psychiatry. 2009;66(6):583-90. 
27. Carrasco M, Volkmar FR, Bloch MH. Pharmacologic treatment of repetitive behaviors in autism spectrum disorders: evidence of publication bias. Pediatrics. 2012;129(5):e1301-10.

This meta-analysis suggests that when unpublished clinical trial data regarding SSRI use for repetitive behaviors in ASD is considered alongside published data, SSRIs do not appear more effective than placebo and may cause significant behavioral side effects.

28. Buitelaar JK, van der Gaag RJ, van der Hoeven J. Buspirone in the management of anxiety and irritability in children with pervasive developmental disorders: results of an open-label study. J Clin Psychiatry. 1998;59(2):56-9.

29. Posey DJ et al. A naturalistic open-label study of mirtazapine in autistic and other pervasive developmental disorders. J Child Adolesc Psychopharmacol 2001;11(3):267-77.

30. Albertini G et al. Compulsive masturbation in infantile autism treated by mirtazapine. Pediatr Neurol. 2006;34(5):417-8.

31. Coskun $\mathrm{M}$ et al. Effectiveness of mirtazapine in the treatment of inappropriate sexual behaviors in individuals with autistic disorder. J Child Adolesc Psychopharmacol. 2009;19(2):203-6.

32. Coskun M, Mukaddes NM. Mirtazapine treatment in a subject with autistic disorder and fetishism. J Child Adolesc Psychopharmacol. 2008;18(2):206-9.

33. Nguyen M, Murphy T. Mirtazapine for excessive masturbation in an adolescent with autism. J Am Acad Child Adolesc Psychiatry. 2001;40(8):8689.

34. Murray MJ. Attention-deficit/hyperactivity disorder in the context of autism spectrum disorders. Curr Psychiatry Rep. 2010;12(5):382-8.

35. Greenhill LL et al. Impairment and deportment responses to different methylphenidate doses in children with ADHD: the MTA titration trial. J Am Acad Child Adolesc Psychiatry. 2001;40(2):180-7.

36. Network, RUoPPA. Randomized, controlled, crossover trial of methylphenidate in pervasive developmental disorders with hyperactivity. Arch Gen Psychiatry. 2005;62(11):1266-74.

37. Handen BL, Sahl R, Hardan AY. Guanfacine in children with autism and/or intellectual disabilities. J Dev Behav Pediatr. 2008;29(4):303-8.

38. Scahill L et al. A prospective open trial of guanfacine in children with pervasive developmental disorders. J Child Adolesc Psychopharmacol. 2006;16(5):589-98.

39. Arnold LE et al. Atomoxetine for hyperactivity in autism spectrum disorders: placebo-controlled crossover pilot trial. J Am Acad Child Adolesc Psychiatry. 2006;45(10):1196-205.

40. Harfterkamp $\mathrm{M}$ et al. A randomized double-blind study of atomoxetine versus placebo for attentiondeficit/hyperactivity disorder symptoms in children with autism spectrum disorder. J Am Acad Child Adolesc Psychiatry. 2012;51(7):733-41.
41. Charnsil C. Efficacy of atomoxetine in children with severe autistic disorders and symptoms of ADHD: an open-label study. J Atten Disord. 2011;15(8):684-9.

42. Posey DJ et al. Open-label atomoxetine for attentiondeficit/ hyperactivity disorder symptoms associated with high-functioning pervasive developmental disorders. J Child Adolesc Psychopharmacol. 2006;16(5):599-610.

43. Marcus RN et al. A placebo-controlled, fixed-dose study of aripiprazole in children and adolescents with irritability associated with autistic disorder. J Am Acad Child Adolesc Psychiatry. 2009;48(11):1110-9.

44. McCracken JT et al. Risperidone in children with autism and serious behavioral problems. N Engl J Med. 2002;347(5):314-21.

45. Owen R et al. Aripiprazole in the treatment of irritability in children and adolescents with autistic disorder. Pediatrics. 2009;124(6):1533-40.

46. Shea $\mathrm{S}$ et al. Risperidone in the treatment of disruptive behavioral symptoms in children with autistic and other pervasive developmental disorders. Pediatrics. 2004;114(5):e634-41.

47. Perrin JM et al. Complementary and alternative medicine use in a large pediatric autism sample. Pediatrics. 2012;130 Suppl 2:S77-82.

48. Valicenti-McDermott M., et al. Use of complementary and alternative medicine in children with autism and other developmental disabilities: associations with ethnicity, child comorbid symptoms, and parental stress. J Child Neurol. 2013.

49. Lofthouse $\mathrm{N}$ et al. A review of complementary and alternative treatments for autism spectrum disorders. Autism Res Treat. 2012;2012:870391.

This paper presents a comprehensive review of CAM treatments commonly used in ASD and rates the strength of evidence for or against their use.

50. Williams K, Wray JA, Wheeler DM. Intravenous secretin for autism spectrum disorders (ASD). Cochrane Database Syst Rev. 2012;4:CD003495.

51. Rossignol DA, Frye RE. Melatonin in autism spectrum disorders: a systematic review and meta-analysis. Dev Med Child Neurol. 2011;53(9):783-92.

52. Bent $\mathrm{S}$ et al. Brief report: hyperbaric oxygen therapy (HBOT) in children with autism spectrum disorder: a clinical trial. J Autism Dev Disord. 2012;42(6):112732.

53. Rossignol DA et al. Hyperbaric treatment for children with autism: a multicenter, randomized, double-blind, controlled trial. BMC Pediatr. 2009;9:21.

54. Granpeesheh D et al. Randomized trial of hyperbaric oxygen therapy for children with autism. Res Autism Spectr Disord. 2010;4(2):268-75.

55. Sampanthavivat $M$ et al. Hyperbaric oxygen in the treatment of childhood autism: a randomised controlled trial. Diving Hyperb Med. 2012;42(3):128-33.

56. Mitka M. CHelation therapy trials halted. JAMA. 2008;300(19):2236. 
57. Baxter AJ, Krenzelok EP. Pediatric fatality secondary to EDTA chelation. Clin Toxicol (Phila). 2008;46(10):1083-4.

58. Brown MJ et al. Deaths resulting from hypocalcemia after administration of edetate disodium: 2003-2005. Pediatrics. 2006;118(2):e534-6.

59. DeJong H, Bunton P, Hare DJ. A systematic review of interventions used to treat catatonic symptoms in people with autistic spectrum disorders. J Autism Dev Disord. 2014;44(9):2127-36.

60. Flippin M, Reszka S, Watson LR. Effectiveness of the picture exchange communication system (PECS) on communication and speech for children with autism spectrum disorders: a meta-analysis. Am J Speech Lang Pathol. 2010;19(2):178-95.

61. Maglione MA et al. Nonmedical interventions for children with ASD: recommended guidelines and further research needs. Pediatrics. 2012;130 Suppl 2:S169-78.

62. Kasari $\mathrm{C}$ et al. Communication interventions for minimally verbal children with autism: a sequential multiple assignment randomized trial. J Am Acad Child Adolesc Psychiatry. 2014;53(6):635-46.

63. Ingersoll B et al. The effects of a developmental, socialpragmatic language intervention on rate of expressive language production in young children with autistic spectrum disorders. Focus Autism Other Dev Disabil. 2005;20(4):213-22.

64. Adams $\mathrm{C}$ et al. The social communication intervention project: a randomized controlled trial of the effectiveness of speech and language therapy for school-age children who have pragmatic and social communication problems with or without autism spectrum disorder. Int J Lang Commun Disord. 2012;47(3):233-44.

65. Provost B, Lopez BR, Heimerl S. A comparison of motor delays in young children: autism spectrum disorder, developmental delay, and developmental concerns. J Autism Dev Disord. 2007;37(2):321-8.

66. Liu T. Motor milestone development in young children with autism spectrum disorders: an exploratory study. Educ Psychol Pract. 2012;28(3):315-26.

67. Clark GJF, Schlabach TL. Systematic review of occupational therapy interventions to improve cognitive development in children ages birth-5 years. Am J Occup Ther. 2013;67(4):425-30.

68. Lang R et al. Sensory integration therapy for autism spectrum disorders: a systematic review. Res Autism Spectr Disord. 2012;6(3):1004-18.

69. Schaaf RC et al. Occupational therapy and sensory integration for children with autism: a feasibility, safety, acceptability and fidelity study. Autism. 2012;16(3):321-7.

70. Lovaas OI, Smith T. Early and intensive behavioral intervention in autism. In: Kazdin AE, Weisz JR, editors. Evidence-based psychotherapies for children and adolescents. New York: Guilford; 2003. p. 325-40.

71. Stevens-Long J., Lovaas OI. Research and treatment with autistic children in a program of behavior therapy. In: Child personality and psychopathology: current topics. Wiley: Oxford; 1974.
72. Remington B et al. Early intensive behavioral intervention: outcomes for children with autism and their parents after two years. Am J Ment Retard.

2007;112(6):418-38.

73. Granpeesheh D, Tarbox J, Dixon DR. Applied behavior analytic interventions for children with autism: a description and review of treatment research. Ann Clin Psychiatry. 2009;21(3):162-73.

74. Koegel RL, Koegel LK, Vernon TW, Brookman-Frazee LI. Empirically supported pivotal response treatment for children with autism spectrum disorders. In: Weisz JR, Kazdin AE, editors. Evidence-based psychotherapies for children and adolescents. 2nd ed. New York: Guilford; 2010. p. 327-44.

75. Koegel LK, Koegel BL, Koegel RL, Vernon TW. Pivotal response treatment. In: Luiselli JK, editor. Children and youth with autism spectrum disorder (ASD): recent advances and innovations in assessment, education, and intervention. New York: Oxford University Press; 2014. p. 134-44.

76. Greenspan SI, Wieder S. Engaging autism: using the floortime approach to help children relate, communicate, and think. Cambridge: Da Capo; 2006.

77. Greenspan SI, Wieder S. The developmental individual-difference, relationship-based (DIR/Floortime) model approach to autism spectrum disorders. In: Hollander E, Anagnostou E, editors. Clinical manual for the treatment of autism. Arlington: American Psychiatric Publishing; 2007. p. 179-209.

78. Rogers SJ, Dawson G. Early start Denver model for young children with autism: promoting language, learning, and engagement. New York: Guilford; 2010.

79.• Dawson G et al. Randomized, controlled trial of an intervention for toddlers with autism: the early start Denver model. Pediatrics. 2010;125(1):e17-23.

Although ABA has been used for decades to treat individuals with ASD, this article highlights one of the new and emerging evidenced therapies for ASD.

80. Virues-Ortega J, Julio FM, Pastor-Barriuso R. The TEACCH program for children and adults with autism: a meta-analysis of intervention studies. Clin Psychol Rev. 2013;33(8):940-53.

81. Schields J. National Autistic Society EarlyBird Programme: partnership with parents. Autism Int J Res Pract. 2001;5(1):49-56.

82. Wright B, Williams C. Intervention and support for parents and carers of children and young people on the autism spectrum: a resource for trainers., JKP resource materials. London: Jessica Kingsley; 2007.

83. Blackledge JT, Hayes SC. Using acceptance and commitment training in the support of parents of children diagnosed with autism. Child Family Behav Ther. 2006;28(1):1-18.

84. Singh NN et al. Mindful parenting decreases aggression, noncompliance, and self-injury in children with autism. J Emot Behav Disord. 2006;14(3):169-77.

85. Singh NN et al. Training in mindful caregiving transfers to parent-child interactions. J Child Fam Stud. 2010;19(2):167-74. 
86. Aman MG et al. Medication and parent training in children with pervasive developmental disorders and serious behavior problems: results from a randomized clinical trial. J Am Acad Child Adolesc Psychiatry. 2009;48(12):1143-54.

87. Storch EA et al. The effect of cognitive-behavioral therapy versus treatment as usual for anxiety in children with autism spectrum disorders: a randomized, controlled trial. J Am Acad Child Adolesc Psychiatry. 2013;52(2):132-42.

Increasing attention is being paid to the psychological experiences of individuals with ASD, and this article presents data on the implementation of one evidence-based intervention for treating comorbid psychiatric symptoms such as anxiety.

88. Hayes SA, Watson SL. The impact of parenting stress: a meta-analysis of studies comparing the experience of parenting stress in parents of children with and without autism spectrum disorder. J Autism Dev Disord. 2013;43(3):629-42.

This article calls attention to the fact that parents of children with ASD experience substantial stress and highlights the need for clinician attention to this issue in addition to assessing and treating the core symptoms of ASD.

89. Lee GK et al. Health-related quality of life of parents of children with high-functioning autism spectrum disorders. Focus Autism Other Dev Disabil. 2009;24(4):227-39.

90. Koegel RL et al. The personality and family-interaction characteristics of parents of autistic children. J Consult Clin Psychol. 1983;51(5):683-92.

91. Hollander $\mathrm{E}$ et al. Oxytocin infusion reduces repetitive behaviors in adults with autistic and Asperger's disorders. Neuropsychopharmacology. 2003;28(1):193-8.

92. Hollander E et al. Oxytocin increases retention of social cognition in autism. Biol Psychiatry. 2007;61(4):498-503.

93. Guastella AJ., et al. The effects of a course of intranasal oxytocin on social behaviors in youth diagnosed with autism spectrum disorders: a randomized controlled trial. J Child Psychol Psychiatry. 2014.

94. Erickson CA et al. STX209 (arbaclofen) for autism spectrum disorders: an 8-week open-label study. J Autism Dev Disord. 2014;44(4):958-64.

95. Rubenstein JL, Merzenich MM. Model of autism: increased ratio of excitation/inhibition in key neural systems. Genes Brain Behav. 2003;2(5):255-67.

96. Ghaleiha A et al. Memantine as adjunctive treatment to risperidone in children with autistic disorder: a randomized, double-blind, placebo-controlled trial. Int J Neuropsychopharmacol. 2013;16(4):783-9.

97. Hardan AY et al. A randomized controlled pilot trial of oral N-acetylcysteine in children with autism. Biol Psychiatry. 2012;71(11):956-61.

98. Ghanizadeh A, Moghimi-Sarani E. A randomized double blind placebo controlled clinical trial of $\mathrm{N}$ acetylcysteine added to risperidone for treating autistic disorders. BMC Psychiatry. 2013;13:196.

99. Eldevik $\mathrm{S}$ et al. Meta-analysis of early intensive behavioral intervention for children with autism. J Clin Child Adolesc Psychol. 2009;38(3):439-50.

100. Johnson CP, Myers SM. Identification and evaluation of children with autism spectrum disorders. Pediatrics. 2007;120(5):1183-215.

101. Volkmar FR, Wiesner LA, Westphal A. Healthcare issues for children on the autism spectrum. Curr Opin Psychiatry. 2006;19(4):361-6.

102. Coury DL et al. Use of psychotropic medication in children and adolescents with autism spectrum disorders. Pediatrics. 2012;130 Suppl 2:S69-76.

This article is also in the aforementioned Supplement to Pediatrics journal. It summarizes the rate of use of psychotropic medications by a large number of individuals enrolled in the Autism Speaks Autism Treatment Network dataset. 